\section{IMPROVING PAEDIATRIC CARDIOLOGY TRANSITION TO ADULT CARDIOLOGY SERVICES IN A DGH, LEVEL 3 CHD CENTRE}

Felicity Cooksey, Cecilia Gonzalez Corcia, Rebecca Lambert, Lucy Duncombe, Gangadhara Bharmappanavara. UK

\subsection{6/bmjpo-2021-RCPCH.217}

Background Transition is the purposeful, planned movement of adolescent and young adults with congenital heart disease to the adult cardiac services. It is not yet an established service across other level three CHD centres. Following a serious incident, a transition clinic for paediatric cardiology patients with complex congenital heart disease was set up at our centre in May 2019. This audit was undertaken to review the existing transition and transfer process of paediatric cardiology patients to adult cardiology services.

Objectives Our aim was to provide an overview of current transitional care for patients with $\mathrm{CHD}$ and to evaluate the process to aid successful transition from paediatric to adult cardiology services according to NICE Standards.

Methods Patients aged 16 to 20 years who had been under paediatric cardiology services at our centre were included. Electronic notes were reviewed retrospectively in October 2020. Current set up has two transition clinics per year attended by a PEC, Paediatric cardiologist and a dedicated ACHD nurse specialist to support. Data was collected on diagnosis, whether under paediatric or adult cardiology services, route of referral, whether patient had been reviewed by adult services or they did not attend the appointment.

Results There were 106 patients aged 16 to 20 years under the care of paediatric cardiology services. Of these, 58 patients $(55 \%)$ were under the care of paediatric services. 48 patients $(45 \%)$ had been referred to adult cardiology services.

$31(65 \%)$ patients were referred to adult services following a standard paediatric clinic appointment including 4 patients with complex CHD. $3(6 \%)$ patients were referred directly with a letter without a paediatric clinic appointment. 14 (29\%) patients were referred via transition clinic. Overall, $14 \%$ (15) patients of 16-to-20-year olds had been reviewed in transition clinic. 3 patients (16\%) did not attend appointments with adult services following transition. These patients were not seen in transition clinic prior to transfer.

Conclusions The majority of patients were seen in a paediatric cardiology clinic to discuss transfer process to adult services, even if not in a formal transition clinic. It is vital to educate adolescent patients with CHD to take charge of their health with a structured transition programme. The DNA rate of patients following transfer to adult services is a potential cause for concern and we need a robust policy in place to address this issue. It is important to determine patients are being given appropriate information and support and we recommend undertaking a patient survey. In long-term, we plan to expand and offer the transition clinic service to CHD patients as per the NICE standard.

\section{LIPID OVERDOSE IN A PRETERM NEONATE: LESSONS LEARNT FROM MEDICATION ERROR}

Sonal Datir, Charlotte Sewsarran, Gopa Sarkar. UK
Background Medication errors can cause significant harm, but are a preventable cause of morbidity and mortality in the presence of effective intervention strategies. The complexity of intravenous medication administration in neonates involves an increased risk of medication errors. Also, neonates have a less capacity to buffer the unintended consequences of the medication error due to physiological immaturity.

Lipids are considered as high alert medication and overdose can cause significant complications including hypertriglyceridemia, respiratory failure, metabolic acidosis, hemolysis, liver dysfunction and pancreatitis. Long-term complications include pulmonary hypertension, chronic lung disease and neurodevelopmental delay.

Objectives Aim: To highlight intervention strategies and learning involved in a medication error due to lipid overdose.

Case History: A baby boy born at 29 weeks was commenced on Parenteral Nutrition (PN) including lipid for suspected Necrotising enterocolitis. The infusions were 'checked' by two trained nurses at the start and at two handovers; with hourly pump readings. After 16 hrs, the infusion pump delivering lipid alarmed noting the bag as empty. This prompted a review of fluid balance chart only to note that the infusion rate of lipids was set incorrectly; $120 \mathrm{mls}$ of lipid was infused; instead of expected $17.1 \mathrm{mls}$ (7 times higher).

The baby developed mild respiratory distress, observations were stable. The lipid infusion was stopped immediately. The triglyceride level of the baby was $83.8 \mathrm{mmol} / \mathrm{l}$ (40 times higher) (Normal $0.34-2.0 \mathrm{mmol} / \mathrm{l}$ ).

The baby was transferred to tertiary NICU, required respiratory support and received 'Double volume exchange transfusion'.

Parents were updated and supported throughout.

Methods INTERVENTIONS: National Patient Safety Agency (NPSA) alert was raised.

A serious incident root-cause analysis was carried out to identify the opportunities to minimise the recurrence of error. This case illustrated a lack of robust checking system and no clearly identifiable process to differentiate between multiple infusions. This emphasised on independent checks by two trained nurses, and cot side checklist during handover (to be signed by two qualified nurses) to allow checking of pumps and rates to overcome involuntary automaticity.

The process of administration of $\mathrm{PN}$ in neonatal unit was reviewed to include a detailed workflow diagram to identify specific problem areas. The bags were colour coded, clear labels were used and infusions were set to run for a maximum of 4 hours.

A competency based workbook was developed to improve uniformity in practice with regards to administrations of medication including PN.

Debrief session and shared learning was organised for all staff, reinforcing the lessons learnt and incorporating into neonatal mandatory training.

Results Our investigation led to a major change in manufacturing nationwide. Based on recommendations, the volume in lipid bags was reduced from $120 \mathrm{mls}$ to $60 \mathrm{mls}$ and changed to red coloured bags.

\section{Conclusions}

1. Exchange transfusion remains the mainstay of treatment for lipid overdose to prevent acute and delayed complications.

2. Human factors play a crucial role. Identifying human errors and developing robust intervention strategies is challenging but very important. 